\title{
Exercise training using hybrid assistive limb (HAL) lumbar type for locomotive syndrome: a pilot study
}

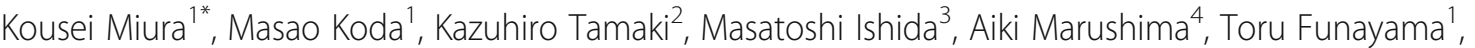 \\ Hiroshi Takahashi ${ }^{1}$, Hiroshi Noguchi ${ }^{1}$, Kentaro Mataki ${ }^{1}$, Yoshihiro Yasunaga ${ }^{1}$, Hiroaki Kawamoto ${ }^{5}$, Yoshiyuki Sankai ${ }^{5}$, \\ Akira Matsumura ${ }^{4,6}$ and Masashi Yamazaki ${ }^{1}$
}

\begin{abstract}
Background: With a rapidly aging population in Japan, locomotive syndrome is becoming an increasingly serious social problem. Exercise therapy using the lumbar type HAL, which is a wearable robot suit that can assist voluntary hip joint motion, would be expected to cause some beneficial effects for people with locomotive syndrome. The purpose of this study was to assess whether the deterioration of low back pain and any other adverse events would occur following HAL exercise therapy. Moreover, the changes of motor ability variables were evaluated.

Methods: We enrolled 33 participants (16 men, 17 women) with locomotive syndrome in this study. They received exercise training (sit-to-stand, lumbar flexion-extension, and gait training) with HAL (in total 12 sessions). We assessed the change of low back pain (lumbar VAS). More than 50\% and $25 \mathrm{~mm}$ increase compared to baseline was defined as adverse events. One-leg standing time (OLST), 10-m walking test (10MWT), Timed Up and Go test (TUG), 1-min sit-to-stand test (1MSTS), FIM mobility scores and EQ-5D were measured.

Results: Of the 33 participants, 32 (16 men, 16 women) (97.0\%) completed all 12 exercise training sessions using the lumbar type HAL. One woman aged 82 years withdrew because of right upper limb pain after the second session regardless of the use of HAL. There was no participant who had deterioration of low back pain. Any other adverse events including external injuries and/or falling, skin disorders, uncontrollable cardiovascular or respiratory disorders, and other health disorders directly related to this exercise therapy did not occur. Several outcome measures of motion ability including OLST, TUG and 1MSTS, EQ VAS and lumbar pain improved significantly after this HAL training.

Conclusions: Almost all patients with locomotive syndrome completed this exercise training protocol without any adverse events related to HAL. Furthermore, balance function variables including OLST, TUG and 1MSTS improved after this HAL exercise therapy even though mobility function variables including 10MWT and FIM mobility scores did not show any significant change. These findings suggest that the exercise therapy using the lumbar type HAL would be one of the options for the intervention in locomotive syndrome.
\end{abstract}

Keywords: Locomotive syndrome, Hybrid assistive limb, Lumbar type, Low back pain, Elderly people

\footnotetext{
*Correspondence: kmiura@tsukuba-seikei.jp

${ }^{1}$ Department of Orthopaedic Surgery, Faculty of Medicine, University of

Tsukuba, 1-1-1 Tennodai, Tsukuba, Ibaraki 305-8575, Japan

Full list of author information is available at the end of the article
}

(C) The Author(s). 2021 Open Access This article is licensed under a Creative Commons Attribution 4.0 International License, which permits use, sharing, adaptation, distribution and reproduction in any medium or format, as long as you give appropriate credit to the original author(s) and the source, provide a link to the Creative Commons licence, and indicate if changes were made. The images or other third party material in this article are included in the article's Creative Commons licence, unless indicated otherwise in a credit line to the material. If material is not included in the article's Creative Commons licence and your intended use is not permitted by statutory regulation or exceeds the permitted use, you will need to obtain permission directly from the copyright holder. To view a copy of this licence, visit http://creativecommons.org/licenses/by/4.0/ The Creative Commons Public Domain Dedication waiver (http://creativecommons.org/publicdomain/zero/1.0/) applies to the data made available in this article, unless otherwise stated in a credit line to the data. 


\section{Background}

There are 34 million elderly people in Japan representing $27 \%$ of the population [1] . With a rapidly aging population in Japan, elderly patients with disorders relating to motor function are becoming an increasingly serious social problem [2]. The Japanese Orthopaedic Association (JOA) proposed the term "locomotive syndrome" in 2007. Locomotive syndrome is defined as a condition in which people have loss of mobility caused by degeneration of locomotive organs and require nursing care services due to disorders related to motor function [3, 4]. In Japanese older than 80 years, the prevalence of locomotive syndrome has reached $60 \%$ in men and $75 \%$ in women [5]. An aging society will be an important issue in the future, not only in Japan, but worldwide [6]. To improve loss of mobility, various exercise therapies have been conventionally performed. More importantly, less invasive therapies such as exercise with the use of robotic-assisted devices are required for elderly people to prevent injury due to excessive physical load.

As for robotic-assisted device for rehabilitation, robotassisted gait training has been recently drawing increasing attention. The Lokomat (Hocoma AG, Volketswil, Switzerland) has been used for the treatment of patients with spinal cord injury and stroke [7]. Some reviews showed the effectiveness of the Lokomat for these diseases $[8,9]$. Although the Lokomat can assist walking movements with the support of body weight, it has the several disadvantages. The Lokomat is not able to assist any movements other than walking and is not portable because of its huge size. Thus, we focused our attention on the exercise therapy by using the lumbar type hybrid assistive limb (HAL) (Cyberdyne Inc., Ibaraki, Japan) (Fig. 1). HAL is the wearable robot suit that can assist joint motion. It can provide voluntary joint motion assist with the reaction to the wearer's intention of standing up by detecting nerve and muscle action potentials of the lumbar erector spinae muscles through electrodes attached on the skin. To date, several types of HAL (HAL for lower limbs and HAL for single joints) have been applied for several musculoskeletal and neurological disorders [10-14]. It has been reported that the lower limb type HAL, which can assist hip and knee joint motion, might improve gait function for myelopathy [10], cerebral infarction [11], and cerebral palsy [12]. The single-joint type HAL can assist knee and elbow joint motion. Several clinical case reports of exercise therapy using the single-joint type HAL for cerebral palsy and brachial plexus injury have been reported [13, 14].

To date, it has been shown the possibility of reduction of the subjective lumbar fatigue and the improvement in lifting performance during repetitive lifting movements [15], repetitive snow-shoveling movements [16], and simulated patient transfer movements [17] in healthy adults with the lumbar type HAL. Moreover, cardiopulmonary burden during stand-up exercise may be reduced because of the use of the lumbar type HAL among healthy volunteers [18]. In addition, the lumbar type HAL is portable and able to assist voluntary joint motion during not only walking but also sit-to-stand and lumbar flexion-extension unlike the Lokomat.

We hypothesized that exercise therapy using the lumbar type HAL would cause some effects on motor
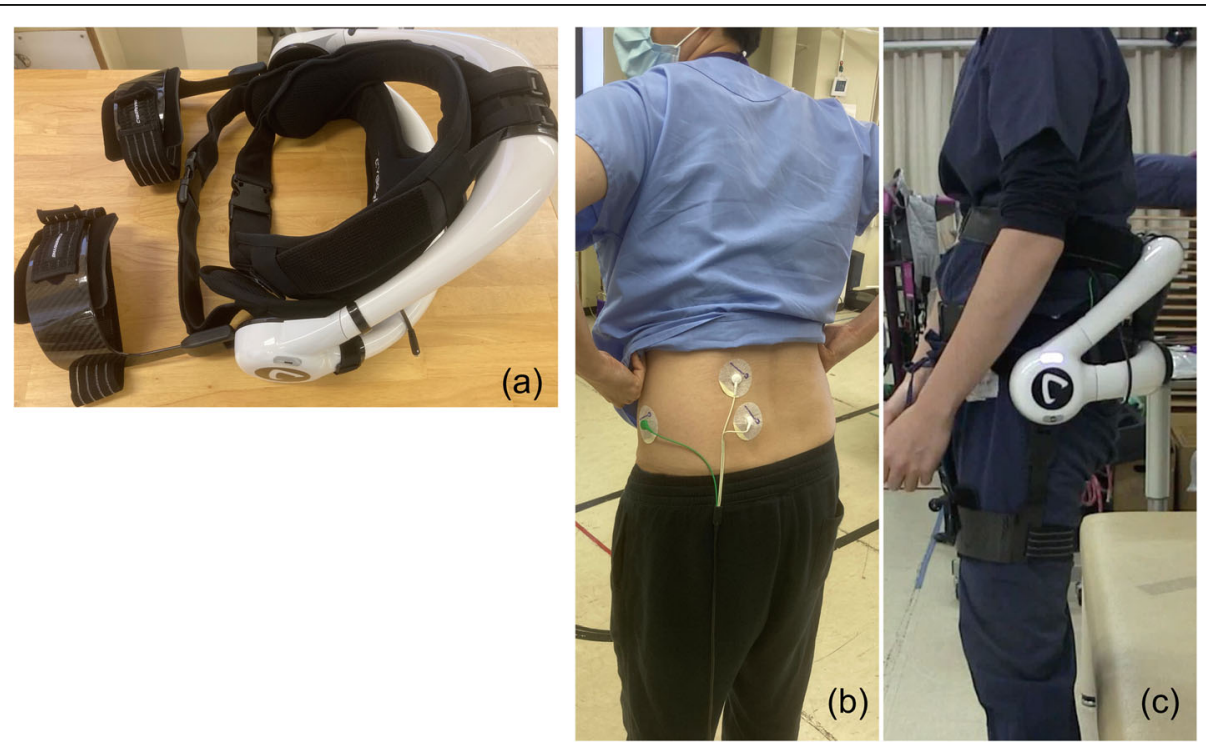

Fig. 1 a Overview of the lumbar type hybrid assistive limb (HAL). b Photograph illustrating electrodes on the skin over lumbar erector spinae muscle to detect muscle action potential. c Lateral image of the worn lumbar type HAL 
function for people with locomotive disorders. However, there are few reports of the use of the lumbar type HAL for those people. The purpose of this pilot study was to assess the low back pain and adverse events following exercise therapy with the lumbar type HAL for safety evaluation. Moreover, we evaluated several outcome measures regarding motor ability and QOL to design advanced clinical trials for ensuring the effects of lumbar type HAL on locomotive syndrome in the future.

\section{Methods}

\section{Participants}

This was a retrospective, single-arm, exploratory study conducted at our institutes between April 2018 and March 2019. The inclusion criteria were as follows: (1) admitted to nursing facilities or the use of day care; (2) aged 40 years or more; (3) at least one affirmative answer on loco-check; (4) suitable body size to fit the lumbar type HAL (maximum waist circumference, $120 \mathrm{~cm}$; maximum thigh circumference, $80 \mathrm{~cm}$ ). Loco-check is a selfassessment questionnaire composed of the following 7 items to evaluate the locomotive syndrome: (1) You cannot put on a pair of socks while standing on one leg; (2) You stumble or slip in your house; (3) You need to use a handrail when going upstairs; (4) You cannot get across the road at a crossing before the traffic light changes; (5) You have difficulty walking continuously for $15 \mathrm{~min}$; (6) You find it difficult to walk home carrying a shopping bag weighing about $2 \mathrm{~kg}$ (e.g., two 1-l milk packs); (7) You find it difficult to do housework requiring physical strength (e.g., use of vacuum cleaner to clean the rooms, putting futons into and taking them out of the closet, etc.) [4]. The exclusion criteria were as follows: (1) skin disorders prohibiting the attachment of the electrodes; (2) inadequately controlled cardiovascular or respiratory disorders interfering with exercise therapy; (3) severe dementia that prohibits understanding of the training program using HAL; (4) lower limb joints disorders that may seriously affect the results of this study.

We obtained informed consent from all participants. This study was approved by the institutional Review Board of our institution (IRB approval No. H29-253) and was performed in accordance with the Declaration of Helsinki.

\section{The lumbar type HAL}

The lumbar type HAL consists of an exoskeletal frame, power units, and lumbar and thigh molds. Actuators of power units, which are located on the wearer's bilateral femoral greater trochanters, generate a torque assisting hip extension motion. A triaxial accelerometer within the exoskeletal frame can detect the absolute trunk angle and angular sensors within the power units and potentiometers can detect the relative angles of the hip joints.
Through these mechanisms, the lumbar type HAL is able to support the wearer's motions with coordination of the level and timing of the torque. Additionally, the lumbar type HAL has two hybrid control systems as follows: a cybernic voluntary control (CVC) and a cybernic autonomous system (CAC). Hara et al. [19] reported these two control systems in detail. CVC system can control the actuator torque of the HAL to augment joint torque of the wearer according to voluntary muscle activity by detecting wearer's motion through myoelectricity. On the other hand, CAC system can support the wearer's weight for reducing moment caused by trunk flexion as a gravity compensation algorithm.

Because of simpler construction compared to the lower limb type HAL, the lumbar type HAL has the advantage of lightness, weighing only $2.9 \mathrm{~kg}$ including the battery. Besides, wearers can equip the lumbar type HAL by themselves in about $5 \mathrm{~min}$.

\section{Training program}

Exercise training with the lumbar type HAL comprised sit-to-stand training, lumbar flexion-extension training, and gait training. Each about 5 min were needed for fitting and removal of the lumbar type HAL. Repetitive sitto-stand training with the lumbar type HAL was performed for $10 \mathrm{~min}$ (Fig. 2). Participants were allowed to push themselves with their hands to stand up if they had difficulty standing up without any assistance. After that, repetitive lumbar flexion and extension motion was performed for $5 \mathrm{~min}$ in a sitting position (Fig. 3). Finally, gait training on the ground was performed continuously using the lumbar type HAL at a pace comfortable for the participant for $5 \mathrm{~min}$ (Fig. 4). If necessary, participants were allowed to use a cane or a walker during gait training. A rest time of $5 \mathrm{~min}$ was set between each training. Each training combined sit-to-stand, lumbar flexion-extension and gait training 3 times per week for 4 weeks (a total of 12 sessions).

\section{Outcome measures}

A retrospective analysis of prospectively collected data was performed. We assessed the change of low back pain by using visual analogue scale (VAS) (lumbar VAS). More than $50 \%$ and $25 \mathrm{~mm}$ increase compared to baseline was defined as adverse events. Moreover, we also defined newly development of the following health disorders related to exercise with the lumbar type HAL as adverse events: (1) external injuries and/or falling during exercise therapy; (2) skin disorders related to wearing HAL; (3) uncontrollable cardiovascular or respiratory disorders interfering with exercise therapy; (4) any other health disorder directly related to this exercise therapy.

Motor ability and quality of life (QOL) were also evaluated at baseline within 1 week before HAL training 


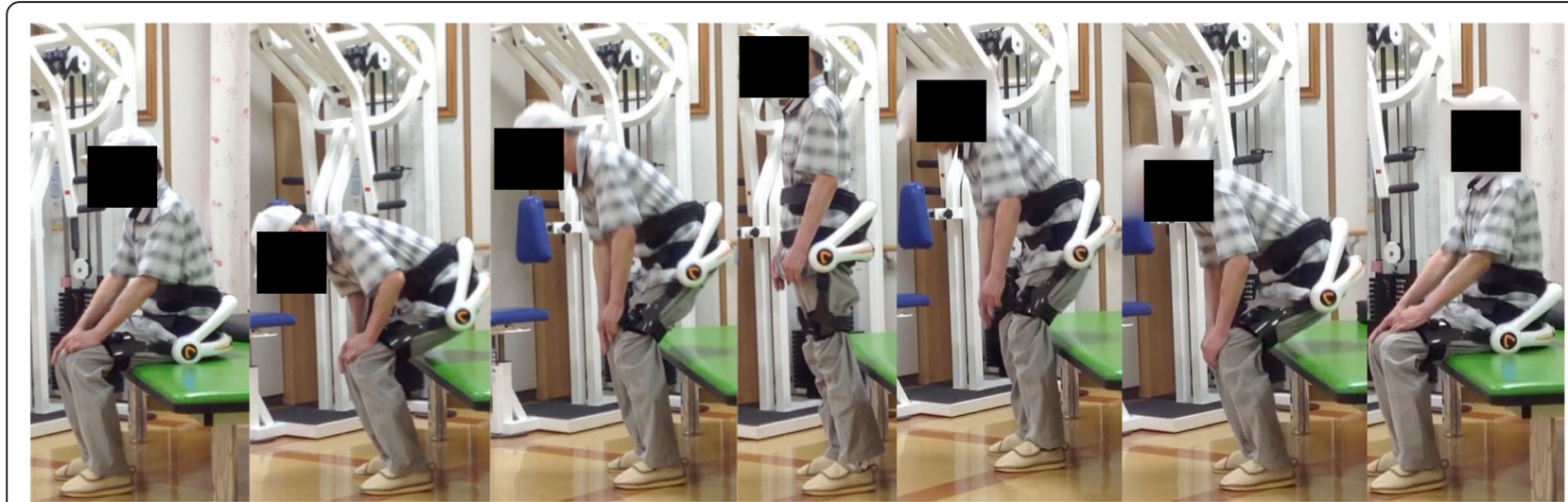

Fig. 2 Repetitive sit-to-stand training with the lumbar type HAL

(pre-HAL) and post-intervention within 1 week after HAL training (post-HAL). Motor ability was assessed with a one-leg standing time (OLST), a 10-m walking test (10MWT), a Timed Up and Go test (TUG), 1min sit-to-stand test (1MSTS), mobility scores (locomotion and stair climb) from the Functional Independence Measure (FIM) assessment tool, and the number of affirmative answers on loco-check. As for OLST, we calculated the average of both sides of lower limb. OLST, TUG and 1MSTS was evaluated only one time without the lumbar type HAL. QOL was assessed using the EuroQOL-5d (EQ-5D) and EQ-Visual Analog Scale (EQ-VAS) [20]. Besides, limb pain was evaluated as the pain in locomotive organ (limb VAS).

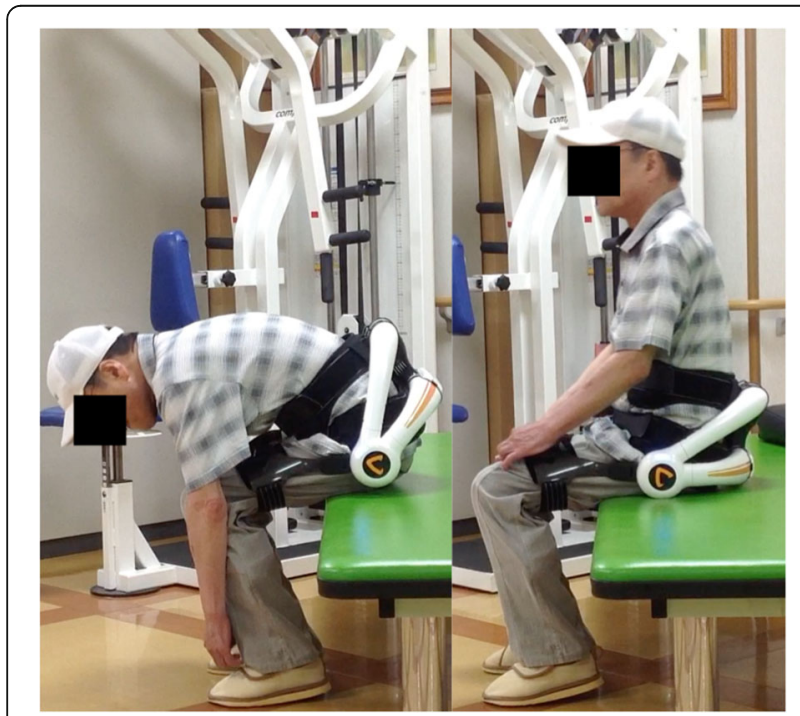

Fig. 3 Repetitive lumbar flexion and extension training with the lumbar type HAL

\section{Statistical analysis}

Some missing data were replaced by baseline observations carried forward. A repeated measures ANOVA was used to evaluate the differences between pre- and postHAL exercise training. All statistical analyses were conducted using the JMP software package ver. 14.0.0 (SAS Institute., Cary, NC, USA), and for all comparisons $P<$ 0.05 was considered a significant difference.

\section{Results}

We prospectively enrolled 33 participants (16 men, 17 women) with locomotive syndrome in the present study. Their mean age \pm SD was $77 \pm 10$ years (range, $40-95$ y), their mean height \pm SD was $155 \pm 7.6 \mathrm{~cm}(140-178 \mathrm{~cm})$,

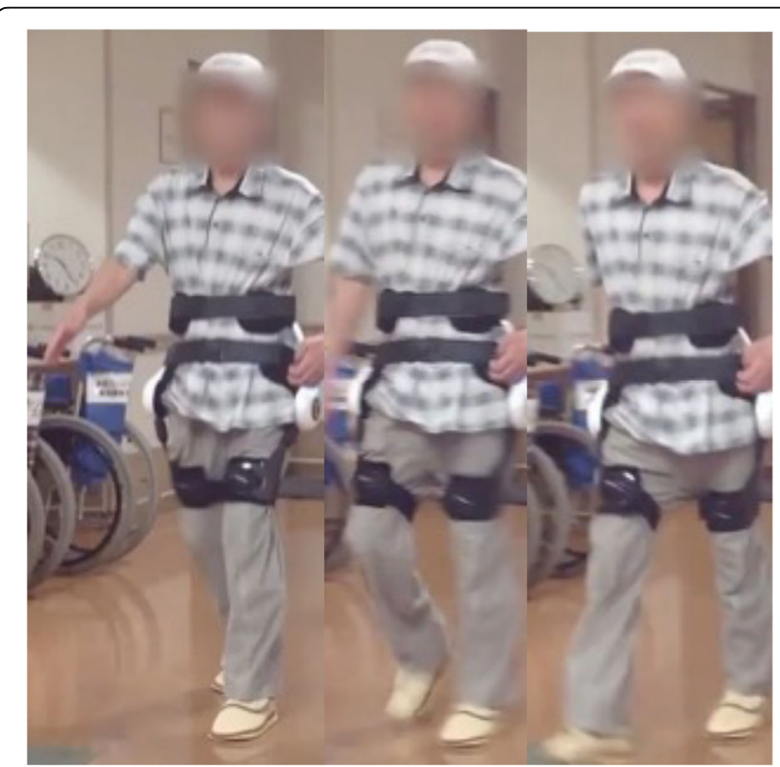

Fig. 4 Continuous gait training on the ground with the lumbar type HA 
and their weight \pm SD was $55 \pm 9.6 \mathrm{~kg}(39-78 \mathrm{~kg})$. The medical comorbidities affecting locomotive function were lumbar spondylosis in 13 cases, cerebrovascular disease in 12 cases, lumbar canal stenosis in 8 case, osteoarthritis of the knee in 7 cases, osteoporosis in 7 cases, osteoporotic vertebral fracture in 5 cases, parkinsonism in 3 cases, proximal femoral fracture in 2 cases, cervical spondylotic myelopathy in 1 case, and spinal cord injury in 1 case.

Of the 33 participants, 32 (16 men, 16 women) (97.0\%) completed all 12 exercise training sessions using the lumbar type HAL. One woman aged 82 years withdrew because of right upper limb pain after the second session. Her exercise therapy was interrupted because she hoped to quit it. Her right upper limb pain was diagnosed as muscular pain and it was spontaneously relieved without any intervention. She needed a walker to walk. Excessive load by walking exercise regardless of the use of HAL on her upper limb to bring the walker might cause her upper limb pain. Of 32 participants that completed the exercise training with HAL, there was no participant who had deterioration of low back pain. Any other adverse events including external injuries and/or falling, skin disorders, uncontrollable cardiovascular or respiratory disorders, and other health disorders directly related to this exercise therapy did not occur.

The comparison of outcome measures is summarized in Table 1. Regarding motor ability, OLST significantly increased after HAL training and exhibited medium effect size $(d=0.76)$. TUG time significantly decreased after HAL training and showed very large effect size $(\mathrm{d}=0.96)$. 1MSTS exhibited significant increase and very large effect size $(d=0.99)$. The number of affirmative answers on loco-check significantly decreased after HAL training and showed medium effect size. The answers of participants to loco-check are summarized in Table 2.
As a result, 13 participants (40.6\%) changed their answers from 'Yes' to 'No' on one or more loco-check questionnaire items. On the other hand, 10MWT and in FIM mobility score did not differ significantly. As for QOL, EQ-VAS significantly increased after HAL training and showed medium effect size $(\mathrm{d}=0.53)$. However, EQ$5 \mathrm{D}$ did not present significant change. Lumbar VAS significantly improved after HAL training and exhibited large effect size $(\mathrm{d}=0.83)$, while limb VAS did not change significantly.

\section{Discussion}

This study showed that 32 of 33 (97.0\%) participants with locomotive syndrome completed exercise therapy with the use of the lumbar type HAL except for one 82year-old woman who hoped to quit it due to upper limb pain after the second session regardless of the use of the HAL. The deterioration of low back pain and any other adverse events did not happen in all participants who completed this exercise therapy. However, there are several patients with locomotive syndrome needed help to equip the lumbar type HAL despite that healthy people can equip by themselves. It would be preferable that it should become easy-to-wear for locomotive syndrome. In addition, several motor function variables including OLST, TUG, 1MSTS improved after HAL training. In particular, 1MSTS and TUG time showed very large effect size. On the other hand, no significant difference was observed in 10MWT and FIM mobility scores. FIM mobility scores are composed of 50-m walking ability and stair climb ability. Thus, FIM mobility scores and 10MWT have been recognized as the evaluation tools of mobility function [21, 22]. On the other hand, OLST, TUG, 1MSTS have been recognized as the evaluation tools of balance function [23, 24]. Therefore, it is suspected that this exercise therapy using the lumbar type

Table 1 Comparison of outcome measures between pre- and post-HAL exercise training $(n=32)$

\begin{tabular}{|c|c|c|c|c|c|c|}
\hline \multirow[b]{2}{*}{ Outcome measures } & & \multicolumn{2}{|l|}{ Pre-HAL } & \multicolumn{3}{|l|}{ Post-HAL } \\
\hline & & Mean \pm SD & $95 \% \mathrm{Cl}$ & Mean \pm SD & $95 \% \mathrm{Cl}$ & Effect size \\
\hline \multirow[t]{6}{*}{ Motor ability } & OLST (s) & $3.8 \pm 6.7$ & $1.13-6.39$ & $6.9 \pm 11.5^{*}$ & $2.31-11.4$ & 0.76 \\
\hline & 10MWT (s) & $15.2 \pm 8.0$ & $11.9-18.5$ & $14.9 \pm 9.1$ & $11.1-18.6$ & 0.06 \\
\hline & TUG (s) & $20.7 \pm 9.3$ & $17.2-24.2$ & $17.7 \pm 7.2^{\dagger}$ & $15.1-20.4$ & 0.96 \\
\hline & 1MSTS (times) & $15.1 \pm 6.5$ & $12.7-17.6$ & $18.0 \pm 6.0^{\dagger}$ & $15.7-20.2$ & 0.99 \\
\hline & FIM mobility scores & $11.0 \pm 2.6$ & $10.1-11.9$ & $11.5 \pm 2.6$ & $10.5-12.4$ & 0.05 \\
\hline & The number of affirmative answers on loco-check & $4.6 \pm 1.7$ & $4.0-5.2$ & $4.2 \pm 1.7^{*}$ & $3.6-4.7$ & 0.71 \\
\hline \multirow[t]{2}{*}{ QOL } & EQ5D & $0.78 \pm 0.11$ & $0.73-0.82$ & $0.80 \pm 0.12$ & $0.76-0.85$ & 0.41 \\
\hline & EQ VAS & $46.4 \pm 18.4$ & $39.8-53.0$ & $59.3 \pm 22.2^{*}$ & $51.9-67.9$ & 0.53 \\
\hline \multirow[t]{2}{*}{ Pain in locomotive Organs } & Lumbar VAS & $33.1 \pm 31.5$ & $21.8-44.2$ & $24.9 \pm 28.6^{*}$ & $14.8-35.1$ & 0.83 \\
\hline & Limb VAS & $32.4 \pm 26.3$ & $23.1-41.8$ & $26.6 \pm 22.5$ & $18.6-34.6$ & 0.46 \\
\hline
\end{tabular}

OLST one-leg standing time, 10MWT 10-m walking test, TUG timed up and go test, 1MSTS 1-min sit-to-stand test, EQ5D EuroQOL-5d ${ }^{*} P<0.05,+P<0.01$ 
Table 2 Patricipants' answers to Loco-check between pre- and post-HAL exercise training

\begin{tabular}{|c|c|c|c|c|}
\hline \multirow[t]{2}{*}{ Loco-check } & \multicolumn{2}{|c|}{ Pre-HAL } & \multicolumn{2}{|c|}{ Post-HAL } \\
\hline & Yes & No & Yes & No \\
\hline (1) You cannnot put on a pair of socks while standing on one leg & 30 & 2 & 30 & 2 \\
\hline (2) You stumble or slip in your house & 12 & 20 & 7 & 25 \\
\hline (3) You need to use a handrail when going upstairs & 31 & 1 & 30 & 2 \\
\hline (4) You cannot get across the road at a crossing before the traffic light changes & 22 & 10 & 21 & 11 \\
\hline (5) You have difficulty walking continuously for $15 \mathrm{~min}$ & 22 & 10 & 20 & 12 \\
\hline (6) You find it difficult to walk home carrying a shopping bag weighing about $2 \mathrm{~kg}$ & 18 & 14 & 13 & 19 \\
\hline (7) You find it difficult to do housework requiring physical strength & 11 & 21 & 9 & 23 \\
\hline
\end{tabular}

HAL may be more helpful for improving the balance function than for improving the mobility function in locomotive syndrome. Regarding lower limb type HAL, it has been reported that walking balance improved after gait training with it [25]. Similarly, repetitive sit-to-stand training and repetitive lumbar flexion and extension training with the use of lumbar type HAL are believed to improve balance function. The lumbar type HAL can provide voluntary hip joint motion assist with the reaction to the wearer's intention of standing up. The characteristic may cause the effect on balance function. However, the detailed mechanisms of that improvement are unclear. Further work such as kinematics analysis is needed to explore the mechanism.

Regarding conventional exercise therapy for locomotive syndrome, other studies have investigated the effects of one-leg stands training and squats training on the elderly. These are considered to be safe and feasible exercises at home [26].

Aoki et al. reported that 87 of 97 elderly participants (89.7\%) completed the exercise training (one-leg stands and squats training) performed for 3 months, and physical function such as OLST and 5 times sit-to-stand tests and 7 of 8 scores of the SF-8 improved significantly [27]. Ishibashi et al. reported that 97 of 151 elderly women (64.2\%) completed one-leg stands and squats training for 2 months, and physical function including OLST, $10 \mathrm{~m}$ maximal gait speed, and knee extension torque improved significantly [28]. Hashimoto et al. reported that 55 of 60 elderly people (91.7\%) completed one-leg stands and squats training for 3 months, and OLST improved significantly [29]. Regarding exercise therapy for locomotive syndrome, it has been reported that gradual increase of the exercise load for people with chronic locomotive degeneration is important for safety [30]. Nevertheless, a high load is required for any functional improvement [26]. Thus, delicate adjustments of exercise load should be considered while exercise training for locomotive syndrome.

Recent advance in robotic-assisted device for rehabilitation including HAL has been remarked. To our best knowledge, a few clinical studies have reported on the robotic-assisted exercise therapy for locomotive syndrome. Kotani et al. [31] firstly reported core exercise and squat exercise using the lumbar type HAL showed significant improvement of motor function in 16 frailty patients including 8 Parkinson's disease (PD) patients and 8 non-PD patients with spine problems. In this study, three kinds of training including sit-to-stand training, lumbar flexion-extension training, and gait training were performed by using the lumbar type HAL for 33 locomotive syndrome patients. Additionally, QOL was assessed in addition to motor function and pain. The completion rate of the exercise therapy with the use of lumbar type HAL was relatively higher compared to previous reported conventional exercise therapy. All participants who completed the HAL exercise training did not develop any adverse events including the increase of low back pain. Moreover, balance function variables including OLST, TUG and 1MSTS improved after HAL exercise therapy similar to previous studies about conventional exercise therapy. As for pain, low back pain and limb pain were evaluated separately. Only low back pain significantly decreased after HAL training. EQ-VAS also showed significant improvement, which might reflect some positive effects on QOL in participants with locomotive syndrome. Presumably, these results are partly due to the special characteristic of HAL to be able to support the wearer's motions with coordination of the level and timing of the torque by detecting nerve and muscle action potentials of the lumbar elector spinae muscles. The coordinated voluntary joint motion assist with the wearer's intention by the lumbar type HAL is considered to induce positive feedback to the wearer's nerve and muscle. We speculate that this novel mechanism of the lumbar type HAL can provide optimal exercise load for locomotive syndrome with loss of mobility. Our results suggest that this exercise training using the lumbar type HAL is promising for locomotive syndrome.

There are several limitations to the present study. First, we did not include a control group. The possibility cannot be excluded that only higher-intensity exercise 
could improve even outcome measures requiring higher motor function regardless of the lumbar type HAL. Next, we could not obtain kinematical measures such as motion analysis and electromyographical measures to assess whether the HAL could suitable exercise load to improve motor ability. Thus, the effect suggested by this HAL exercise training for locomotive syndrome should not be generalized. Furthermore, we had no available follow up data. These data are important for evaluating the long-term outcome of this protocol of exercise training using the lumbar type HAL. Further studies on these issues are necessary to clarify the effect of the lumbar type HAL for locomotive syndrome.

\section{Conclusion}

Our results revealed that 32 of 33 (97.0\%) participants with locomotive syndrome completed exercise therapy with the use of the lumbar type HAL. The deterioration of low back pain and any other adverse events did not occur in all participants who completed this exercise therapy. Regarding motor ability, balance function variables including OLST, TUG and 1MSTS improved after this HAL exercise therapy even though mobility function variables including 10MWT and FIM mobility scores did not show any significant change. Furthermore, lumbar VAS and EQ-5D significantly improved after exercise therapy with HAL. These findings suggest that the exercise therapy using the lumbar type HAL would be one of the options for the intervention in locomotive syndrome. Further studies are needed to elucidate the effect of the exercise therapy using the lumbar type HAL for locomotive syndrome.

\section{Abbreviations \\ HAL: Hybrid assistive limb; OLST: One-leg standing time; 10MWT: 10-m walking test; TUG: Timed up and go test; 1MSTS: 1-min sit-to-stand test; JOA: Japanese Orthopaedic Association; CVC: Cybernic voluntary control; CAC: Cybernic autonomous system; QOL: Quality of life; FIM: Functional independence measure; EQ-5D: EuroQOL-5d; EQ-VAS: EQ-visual analog scale}

\section{Acknowledgements}

Not applicable.

\section{Authors' contributions}

KM and MK participated in the design of this study, performed the statistical analysis and drafted the manuscript under supervision of AM (Akira Matsumura) and MY. KT and MI recruited participants. AM (Aiki Marushima), TF, HT, HN and KM helped to draft the manuscript and revised it. YY, HK, YS supervised the research project. All authors read and approved the final manuscript.

\section{Funding}

The work was supported by ImPACT Program of Council for Science, Technology and Innovation (Cabinet Office, Government of Japan).

\section{Availability of data and materials}

The datasets generated and/or analysed during the current study are not publicly available due to limitations of ethical approval involving the patient data and anonymity but are available from the corresponding author on reasonable request.

\section{Declarations}

Ethics approval and consent to participate

We obtained informed consent from all participants. This study was approved by the institutional Review Board of Tsukuba University (IRB approval No. H29-253) and was performed in accordance with the Declaration of Helsinki.

\section{Consent for publication}

Patients signed informed consent regarding publishing their data.

\section{Competing interests}

A commercial party having a direct financial interest in the results of the research supporting this article has conferred or will confer a financial benefit on one or more of the authors. YS is a professor of University of Tsukuba, a founder, a shareholder, and the chief executive officer of University venture company "CYBERDYNE Inc.", Ibaraki, Japan. HK is an associate professor of University of Tsukuba, a co-founder, a shareholder, and an outside director of University venture company CYBERDYNE. YY is a Ph.D. student at the University of Tsukuba and the general manager of sales and director of the university's venture company "CYBERDYNE, Inc." in Ibaraki, Japan. COI is managed by University of Tsukuba according to National university rules and guidelines and by board of directors of CYBERDYNE. The patent royalty is paid to University of Tsukuba from CYBERDYNE and a part of the royalty is paid to YS from University of Tsukuba according to National university rules. CYBERDYNE is a R\&D company and the manufacturer of the HAL. The present study was proposed by the authors. CYBERDYNE was not directly involved in the study design; collection, analysis, or interpretation of data; writing the report; or the decision to submit the paper for publication. Other authors report they have no conflict of interest to disclose.

\section{Author details}

${ }^{1}$ Department of Orthopaedic Surgery, Faculty of Medicine, University of Tsukuba, 1-1-1 Tennodai, Tsukuba, Ibaraki 305-8575, Japan. ${ }^{2}$ Medical Corporation, Kanjinkai, 3-35-13, Kamidaira, Fussa, Tokyo 197-0012, Japan. ${ }^{3}$ Eijyu Care Center, 1-2-30, Uriwariminami, Hirano, Osaka 547-0023, Japan. ${ }^{4}$ Department of Neurosurgery, Faculty of Medicine, University of Tsukuba, 1-1-1 Tennodai, Tsukuba, Ibaraki 305-8575, Japan. ${ }^{5}$ Center for Cybernics Research, University of Tsukuba, 1-1-1 Tennodai, Tsukuba, Ibaraki 305-8575, Japan. ${ }^{6}$ Ibaraki Prefectural University of Health Sciences, 4773 Ami,

Inashiki-gun, Ibaraki 300-0331, Japan.

Received: 8 March 2021 Accepted: 1 June 2021

Published online: 12 June 2021

\section{References}

1. Ministry of Health LaW. Long-term care insurance system of Japan. 2016; Available from: https://www.mhlw.go.jp/english/policy/care-welfare/carewelfare-elderly/dl/Itcisj_e.pdf

2. Ishibashi H. Locomotive syndrome in Japan. Osteoporos Sarcopenia. 2018:4(3):86-94.

3. Nakamura K. A "super-aged" society and the "locomotive syndrome". J Orthop Sci. 2008;13(1):1-2.

4. Nakamura K. The concept and treatment of locomotive syndrome: its acceptance and spread in Japan. J Orthop Sci. 2011;16(5):489-91.

5. Yoshimura N, Muraki S, lidaka T, Oka H, Horii C, Kawaguchi H, et al. Prevalence and co-existence of locomotive syndrome, sarcopenia, and frailty: the third survey of research on osteoarthritis/osteoporosis against disability (ROAD) study. J Bone Miner Metab. 2019;37(6):1058-66.

6. United Nations DoEaSA. World population ageing. 2017; Available from: https:/umw.un. org/en/development/desa/population/publications/pdf/ageing/WPA2017_Highlights.pdf

7. Jezernik S, Colombo G, Keller T, Frueh H, Morari M. Robotic orthosis lokomat: a rehabilitation and research tool. Neuromodulation. 2003;6(2):108-15.

8. Nam KY, Kim HJ, Kwon BS, Park JW, Lee HJ, Yoo A. Robot-assisted gait training (Lokomat) improves walking function and activity in people with spinal cord injury: a systematic review. J Neuroeng Rehabil. 2017;14(1):24.

9. Bruni MF, Melegari C, De Cola MC, Bramanti A, Bramanti P, Calabrò RS. What does best evidence tell us about robotic gait rehabilitation in stroke patients: a systematic review and meta-analysis. J Clin Neurosci. 2018;48:11-7.

10. Kubota S, Abe T, Kadone H, Shimizu Y, Funayama T, Watanabe H, et al. Hybrid assistive limb (HAL) treatment for patients with severe thoracic myelopathy due to ossification of the posterior longitudinal ligament 
(OPLL) in the postoperative acute/subacute phase: a clinical trial. J Spinal Cord Med. 2019:42(4):517-25.

11. Watanabe H, Marushima A, Kadone H, Ueno T, Shimizu Y, Kubota S, et al. Effects of gait treatment with a single-leg hybrid assistive limb system after acute stroke: a non-randomized clinical trial. Front Neurosci. 2019;13:1389.

12. Nakagawa S, Mutsuzaki H, Mataki Y, Endo Y, Matsuda M, Yoshikawa K, et al. Safety and immediate effects of hybrid assistive limb in children with cerebral palsy: a pilot study. Brain and Development. 2020;42(2):140-7

13. Kubota S, Kadone H, Shimizu Y, Hara Y, Kubo T, Watanabe H, et al. Robotic rehabilitation training with a newly developed upper limb single-joint Hybrid Assistive Limb (HAL-SJ) for elbow flexor reconstruction after brachial plexus injury: a report of two cases. J Orthop Surg (Hong Kong). 2018;26(2):2309499018777887.

14. Shimizu Y, Kadone H, Kubota S, Ueno T, Sankai Y, Hada Y, et al. Voluntary elbow extension-flexion using single joint hybrid assistive limb (HAL) for patients of spastic cerebral palsy: two cases report. Front Neurol. 2019:10:2.

15. Miura K, Kadone H, Koda M, Abe T, Kumagai H, Nagashima K, et al. The hybrid assistive limb (HAL) for care support successfully reduced lumbar load in repetitive lifting movements. J Clin Neurosci. 2018;53:276-9.

16. Miura K, Kadone H, Koda M, Abe T, Endo H, Murakami H, et al. The hybrid assisted limb (HAL) for care support, a motion assisting robot providing exoskeletal lumbar support, can potentially reduce lumbar load in repetitive snow-shoveling movements. J Clin Neurosci. 2018:49:83-6.

17. Miura K, Kadone H, Abe T, Koda M, Funayama T, Noguchi H, Kumagai $H$ Nagashima K, Mataki K, Shibao Y et al: S uccessful Use of the Hybrid Assistive Limb for Care Support to Reduce Lumbar Load in a Simulated Patient Transfer. Asian Spine J. 2021;15:40-5.

18. Watanabe H, Koike A, Pak YJ, Wu L, Kubota H, Konno H, et al. Effects of a lumbar-type hybrid assistive limb on cardiopulmonary burden during squat exercise in healthy subjects. J Clin Neurosci. 2019;66:226-30.

19. Hara H, Sankai Y. Development of HAL for lumbar support. In: Japan Society for Fuzzy Theory and Intelligent Informatics; 2010. p. 416-21.

20. Group E. EuroQol--a new facility for the measurement of health-related quality of life. Health Policy. 1990;16(3):199-208.

21. Ottenbacher KJ, Hsu Y, Granger CV, Fiedler RC. The reliability of the functional independence measure: a quantitative review. Arch Phys Med Rehabil. 1996;77(12):1226-32.

22. Martin WJ. Refining the ten-metre walking test for use with neurologically impaired people. Physiotherapy. 2002;88:386-97.

23. Borowicz A, Zasadzka E, Gaczkowska A, Gawłowska O, Pawlaczyk M. Assessing gait and balance impairment in elderly residents of nursing homes. J Phys Ther Sci. 2016;28(9):2486-90.

24. Franchignoni F, Tesio L, Martino MT, Ricupero C. Reliability of four simple, quantitative tests of balance and mobility in healthy elderly females. Aging (Milano). 1998;10(1):26-31.

25. Yoshimoto T, Shimizu I, Hiroi Y, Kawaki M, Sato D, Nagasawa M. Feasibility and efficacy of high-speed gait training with a voluntary driven exoskeleton robot for gait and balance dysfunction in patients with chronic stroke: nonrandomized pilot study with concurrent control. Int J Rehabil Res. 2015:38(4):338-43.

26. Nakamura K, Ogata T. Locomotive syndrome: definition and management. Clin Rev Bone Miner Metab. 2016;14:56-67.

27. Aoki K, Sakuma M, Ogisho N, Nakamura K, Chosa E, Endo N. The effects of self-directed home exercise with serial telephone contacts on physical functions and quality of life in elderly people at high risk of locomotor dysfunction. Acta Med Okayama. 2015;69(4):245-53.

28. Ishibashi $H$, Fujita $H$. The effect of locomotion training on mobility function in the elderly female. Osteoporos Jpn. 2011;19(3):391-7 (in Japanese).

29. Hashimoto M, Yasumura S, Nakano K, Kimura M, Nakamura K, Fujino K, et al. Feasibility study of locomotion training in a home-visit preventive care program. Nihon Ronen Igakkai Zasshi. 2012;49(4):476-82 (in Japanese).

30. Yoshimura N, Muraki S, Oka H, Mabuchi A, En-Yo Y, Yoshida M, et al. Prevalence of knee osteoarthritis, lumbar spondylosis, and osteoporosis in Japanese men and women: the research on osteoarthritis/osteoporosis against disability study. J Bone Miner Metab. 2009;27(5):620-8.

31. Kotani N, Morishita T, Yatsugi A, Fujioka S, Kamada S, Shiota E, et al. Biofeedback Core exercise using hybrid assistive limb for physical frailty patients with or without Parkinson's disease. Front Neurol. 2020;11:215.

\section{Publisher's Note}

Springer Nature remains neutral with regard to jurisdictional claims in published maps and institutional affiliations.

Ready to submit your research? Choose BMC and benefit from:

- fast, convenient online submission

- thorough peer review by experienced researchers in your field

- rapid publication on acceptance

- support for research data, including large and complex data types

- gold Open Access which fosters wider collaboration and increased citations

- maximum visibility for your research: over $100 \mathrm{M}$ website views per year

At $\mathrm{BMC}$, research is always in progress.

Learn more biomedcentral.com/submissions 